\title{
Fast switches in relay feedback systems ${ }^{1}$
}

\author{
Karl Henrik Johansson ${ }^{\mathrm{a}}$, Anders Rantzer ${ }^{\mathrm{b}}$,*, Karl Johan Åström ${ }^{\mathrm{b}}$ \\ a Department of Electrical Engineering and Computer Sciences, University of California at Berkeley, Berkeley, CA 94720, USA \\ ${ }^{\mathrm{b}}$ Department of Automatic Control, Lund Institute of Technology, P.O. Box 118, S-221 00 Lund, Sweden \\ Received 22 March 1996; revised 27 April 1998; received in final form 10 September 1998
}

Scalar linear systems with relay feedback are analyzed. In particular, fast relay switches and various types of limit cycles are investigated.

\begin{abstract}
Relays are common in automatic control systems. Even linear systems with relay feedback are, however, far from fully understood. New results are given about the behavior of these systems via a state-space approach. It is proved that there exist multiple fast switches if and only if the sign of the first nonvanishing Markov parameter of the linear system is positive. Fast switches are shown to occur as part of stable limit cycles. An analysis is developed for these limit cycles that illustrates how they can be predicted. (C) 1999 Elssevier Science Ltd. All rights resesrved.
\end{abstract}

Keywords: Relay control; Sliding mode; Limit cycles; Chattering; Nonsmooth differential equations; Oscillations; Piecewise linear systems

\section{Introduction}

Analysis of relay feedback systems is a classical topic in control theory. The early work was motivated by relays in electromechanical systems and simple models for dry friction. The design of relay controllers in aerospace applications gave inspiration to the development of the self-oscillating adaptive controller in the 1960s (FlüggeLotz, 1953, 1968). Recently new interest in relay feedback appeared due to the idea of using relays for tuning simple controllers in Åström and Hägglund (1984b). By simply replacing the controller with a relay, measuring the amplitude and frequency of the possible oscillation, and deriving the controller parameters from these, a robust control design method is obtained. Although this method is now widely used in industry ( $̊$ ström and Hägglund,

\footnotetext{
*Corresponding author. Tel.: + 46462228778 ; fax: + 4646138118 ; e-mail: rantzer@control.lth.se.

${ }^{1}$ An earlier version of this paper was presented at the 13th IFAC World Congress, held in San Francisco, CA, USA, 1-5 July 1996. This paper was recommended for publication in revised form by Associate Editor Hassan Khalil under the direction of Editor T. Başar.
}

1995), there are several issues that need further theoretical analysis. One problem is to characterize those systems that will give a globally stable limit cycle. This problem is important because it gives a class of systems, where relay tuning can be theoretically justified.

The idea to put the plant under relay feedback is also used in other applications. In Smith and Doyle (1993) perturbation bounds are estimated for robust control design and in Lundh and Åström (1994) it is shown how initialization of adaptive controllers can be done. Quantization in digital control can be analyzed with relay feedback methods. Limit cycles due to quantizers are reported in Parker and Hees (1971). Relays are key components in variable-structure systems (Utkin, 1987). More applications of relays in control systems are given in Tsypkin (1984), Åström (1995), and Johansson (1997). The monograph Andronov et al. (1965) is an early classical reference (first edition published in Russian in 1937) discussing oscillations is relay feedback systems using phase-plane analysis.

Analysis of linear systems with relay feedback is a nontrivial task. The major reference about relay control systems Tsypkin (1984) surveys a number of analysis 
methods and results. In particular, it discusses the earlier work Anosov (1959), which relates the stability properties of relay feedback to those of high gain linear feedback.

Other applicable stability results, valid for a more general class of nonlinearities, are given in Yakubovich (1964). A nonsmooth Lyapunov stability theory is developed in Shevitz and Paden (1994). Relay feedback systems often tend to a limit cycle. Methods for estimating oscillation frequency and amplitude are thoroughly discussed in Tsypkin (1984); see also Atherton (1975) and Mees (1981). It is important to note that all these frequency methods are derived under the assumption that a limit cycle exists. To tell in general if all trajectories of a relay feedback system actually converges to a periodic orbit is an open problem. In Yakubovich (1973) a frequency condition is used to give sufficient conditions for a certain type of oscillations. Holmberg (1991) shows through phase-plane analysis that some first-order and second-order systems have a globally stable limit cycle. Megretski (1996) proves that this also holds for higherorder systems having an impulse response sufficiently close, in a certain sense, to a second-order nonminimum phase system.

Relay feedback systems may exhibit several interesting behaviors. The main contribution of our work is to investigate some of these and state a number of new results to improve the understanding of linear systems with relay feedback. Particular emphasis is on fast switches and their properties. It is shown that a necessary and sufficient condition for multiple fast switches is that the sign of the first nonvanishing Markov parameter is positive. This result can be seen as a generalization of the condition on convergence to a first-order sliding set in relay feedback systems (Filippov, 1988). The condition for multiple fast switches in third-order systems was given in Johansson and Rantzer (1996). Here, the condition is generalized to systems of arbitrary order. An application of the result is to predict fast switches as part of limit cycles. This is done in the latter part of the paper, where it is also shown how these complicated limit cycles can be analyzed using Utkin's equivalent control (Utkin, 1987). Further analysis of the limit cycles is done in Johansson (1997) and Johansson et al. (1997).

There exist conditions on local stability of limit cyles in the literature. Two important ones are given in Balasubramanian (1981) and Åström and Hägglund (1984a), respectively. The conditions are rephrased here and it is shown that they are equivalent if the pole excess of the linear system is greater than one.

The outline of the paper is as follows. Some notations and assumptions are given in Section 2. In Section 3 two conditions for local stability of limit cycles are compared. The main result on multiple fast switches is given in Section 4. Some approximate analysis to gain extra insight is done in Section 5. Section 6 presents systems of various pole excesses that exhibit limit cycles with fast switches.

\section{Preliminaries}

Consider a relay feedback system that consists of a linear system $G$ and a relay defined as follows. The system $G$ is a strictly proper transfer function with scalar input $u$ and scalar output $y$. Let a minimal state-space representation of $G$ be given by

$\dot{x}=A x+B u$,

$y=C x$,

where $x=\left(x_{1}, \ldots, x_{n}\right)^{\mathrm{T}} \in \mathbb{R}^{n}$. The relay feedback is defined by

$u=-\operatorname{sgn} y \in \begin{cases}\{-1\}, & y>0, \\ {[-1,1],} & y=0, \\ \{1\}, & y<0 .\end{cases}$

The switch plane $\mathscr{S}$ is the hyperplane of dimension $n-1$ where the output vanishes, that is,

$\mathscr{S}:=\{x: C x=0\}$.

On either side of $\mathscr{S}$ the feedback system is linear: if $C x>0$ the dynamics are given by $\dot{x}=A x-B$, and if $C x<0$ we have $\dot{x}=A x+B$. We also introduce the notation

$S_{+}:=\{x \in \mathscr{S}: C A x-C B>0\}$.

The linear dynamics on each side of $\mathscr{S}$ have fixed points equal $\pm A^{-1} B$ (if $A$ is nonsingular), so positive steadystate gain $-C A^{-1} B>0$ guarantees the trajectories not to tend to any of these two fixed points. This thus ensures a relay switch to occur.

An absolutely continuous function $x:[0, \infty) \mapsto \mathbb{R}^{n}$ is called a trajectory of Eqs. (1)-(2) if it satisfies (1) and (2) almost everywhere. Note that a differential equation with discontinuous right-hand sides may have nonunique trajectories (Filippov, 1988). A limit cycle $\mathscr{L} \subset \mathbb{R}^{n}$ in this paper denotes the set of values attained by a periodic trajectory, which is isolated and not an equilibrium. The limit cycle $\mathscr{L}$ is symmetric if for every $x \in \mathscr{L}$ it is also true that $-x \in \mathscr{L}$. For system (1)-(2), the limit cycle is simple if there exists a trajectory $x$ in $\mathscr{L}$ with period $T$ and a number $\tau \geq T / 2$ such that $C x(t) \geq 0$ for $t \in[\tau, \tau+T / 2]$ and $C x(t) \leq 0$ for $t \in[\tau-T / 2, \tau]$. A limit cycle is then stable if for each $\varepsilon>0$ there exists $\delta>0$ such that $\left|d_{\mathscr{L}}(x(0))\right|<\delta$ implies that $d_{\mathscr{L}}(x(t))<\varepsilon$ for all $t>0$.

Assume that $A$ is stable. The Poincare map $g: \mathscr{S}_{+} \mapsto \mathscr{S}_{+}$for the system (1)-(2) is defined as

$g(x)=-\mathrm{e}^{A h(x)} x+\left(\mathrm{e}^{A h(x)}-I\right) A^{-1} B$,

where $h(x)$ is the switch time, that is, the minimal time that gives $g(x) \in \mathscr{S}_{+}$. 
Intuitively, it seems reasonable to approximate a relay by a saturation with steep slope. This is done in Tsypkin (1984). There are, however, subtleties in the limit as the slope tends to infinity. If this limit is not dealt with properly, erroneous results may be derived. An illustration is given in the discussion on Balasubramanian's stability condition in Section 3.

\section{Stability of limit cycles}

An important behavior of relay feedback systems is that they often have a stable limit cycle. In this section a sufficient condition for local stability of a limit cycle is given. The condition was derived in Åström and Hägglund (1984a) and Åström (1995). It is here compared to a similar result in the literature.

An obvious question is whether there exist relay feedback systems that do not have a unique stable limit cycle. For higher-order systems this is the case, as shown by the following example.

\section{Example 1. Let}

$G(s)=\frac{(s+1)^{2}}{(s+0.1)^{3}(s+7)^{2}}$.

Depending on the initial conditions, the relay feedback system tends to either a slow or a fast limit cycle. In Fig. 1 the relay output $u$ is shown for the two cases after the initial transient has disappeared. Analysis shows that the limit cycles are locally stable, see Example 3. Describing function analysis, see Atherton (1975), gives in this case the correct qualitative result.

If $x^{*} \in \mathscr{S}_{+}$is the switch plane interssection of a stable simple limit cycle, then $x^{*}$ is a fixed point of the Poincare map $g$, that is, $x^{*}=g\left(x^{*}\right)$. Hence, the solutions of the equation $x=g(x)$ give candidates for simple limit cycle intersections with $\mathscr{S}_{+}$. If $A$ is nonsingular, the solution is given by

$x=\left(\mathrm{e}^{A h(x)}+I\right)^{-1}\left(\mathrm{e}^{A h(x)}-I\right) A^{-1} B$.

The following proposition is proved in Åström and Hägglund (1984a) and Åström (1995) by the classical approach of studying small perturbations of the Poincare $\operatorname{map} g$.

Proposition 1. Consider the relay feedback system (1)-(2) with nonsingular $A$. If there exists a simple limit cycle with period $2 h^{*}$, then

$f\left(h^{*}\right):=C\left(\mathrm{e}^{A h^{*}}+I\right)^{-1}\left(\mathrm{e}^{A h^{*}}-I\right) A^{-1} B=0$.
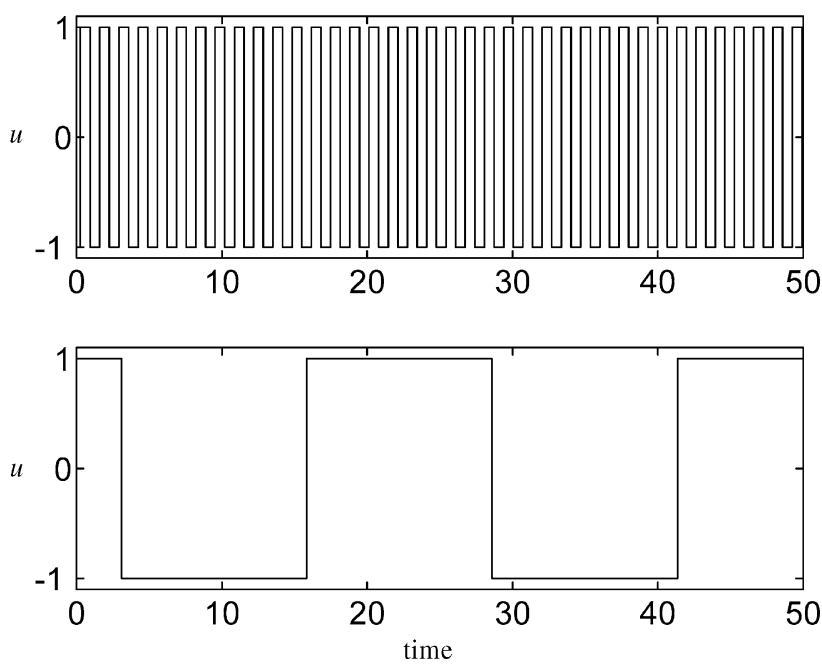

Fig. 1. Two stable limit cycles for the system in Example 1.

The limit cycle is stable if all eigenvalues of

$W_{a}:=\left(I-\frac{w C}{C w}\right) \mathrm{e}^{A h^{*}}, \quad w=2\left(\mathrm{e}^{A h^{*}}+I\right)^{-1} \mathrm{e}^{A h^{*}} B$

are in the open unit disc. The limit cycle is unstable if at least one eigenvalue is outside the unit disc.

Note that $f(0)=0$, so the trivial solution $h^{*}=0$ always satisfies the necessary condition (5). It is easy to show that this is the only solution for first-order systems and for second-order systems with no zeros. Hence, these systems exhibit no simple limit cycles under relay feedback. The assumption that $A$ is nonsingular can be easily removed, (Åström, 1995).

Stability of limit cycles was also studied in Balasubramanian (1981). The relay feedback system is rewritten as a periodically time-varying linear system, which gives the following result.

Proposition 2. Consider the relay feedback system (1)-(2) with $C B=0$. A simple limit cycle with period $2 h^{*}$ is stable if one eigenvalue of

$$
\begin{gathered}
W_{b}:=\exp \left(-\frac{2 B C}{C w}\right) \exp \left(A h^{*}\right), \\
w=2\left(\mathrm{e}^{A h^{*}}+I\right)^{-1} \mathrm{e}^{A h^{*} B}
\end{gathered}
$$

is on the unit circle and the others are in the open unit disc. The limit cycle is unstable if at least one eigenvalue is outside the unit disc.

From Eq. (4) it follows that $C w=C A x^{*}+C B$, where $x^{*} \in \mathscr{S}_{+}$corresponds to the switch plane interssections of the limit cycle. If $C B \neq 0$, the output $y$ possesses a discontinuity at the relay switches. It was suggested in 
Balasubramanian (1981) that a simiar result to Propositison 2 holds for $C B \neq 0$, if $W_{b}$ is replaced by

$\hat{\mathrm{W}}_{b}=\exp \left(B C\left[\left(C \mathrm{e}^{-A h^{*}} w\right)^{-1}-(C w)^{-1}\right]\right) \exp \left(A h^{*}\right)$.

See also Wadey and Atherton (1986) and Atherton (1993). Note that $w$ is the velocity immediately prior to the switch. The expression for $\hat{W}_{b}$ is obtained simply by replacing $(C w)^{-1}$ by the harmonic mean immediately before and after the switches. The criterion is, however, not correct. See the following example.

Example 2. Consider the system

$G(s)=\frac{\beta s+1}{(s+1)(s+2)}$

with state-space representation

$\dot{x}=\left[\begin{array}{ll}0 & -2 \\ 1 & -3\end{array}\right] x+\left[\begin{array}{l}1 \\ \beta\end{array}\right] u$,

$y=\left[\begin{array}{ll}0 & 1\end{array}\right] x$,

and relay feedback. Let $\beta=-1$. Eq. (5) has only one positive solution $h^{*}=1.76$. The eigenvalues of $W_{a}$ are 0 and -0.03 for $h^{*}$, so a locally stable limit cycle is predicted. In contrast, the eigenvalues of $\hat{W}_{b}$ are -0.02 and -31.38 . It is possible to show that the system has a globally stable limit cycle, for example, see Holmberg (1991). Hence, $\hat{W}_{b}$ erroneously predicts an unstable limit cycle.

Next, we show that Propositions 1 and 2 are equivalent if $C B=0$. First, note that if $C B=0$, then

$\exp \left(-\frac{2 B C}{C w}\right)=\sum_{k=0}^{\infty} \frac{(-2)^{k}}{k !(C w)^{k}}(B C)^{k}=I-\frac{2 B C}{C w}$,

so that

$W_{b}=\left(I-\frac{2 B C}{C w}\right) \mathrm{e}^{A h^{*}}$.

Proposition 3. Consider $W_{a}$ and $W_{b}$ as previously defined and assume $C B=0$. Then, $W_{a}$ has one eigenvalue equal to 0 and $W_{b}$ has one eigenvalue equal to -1 . Furthermore, $\lambda \notin\{-1,0\}$ is an eigenvalue of $W_{a}$ if and only if $\lambda$ is also an eigenvalue of $W_{b}$.

Proof. Combining Eqs. (6) and (7), straightforward calculations give

$W_{b}=W_{a}-\mathrm{e}^{-A h^{*}} \frac{w C}{C w} \mathrm{e}^{A h^{*}}$.

From the equalities

$W_{a} \mathrm{e}^{-A h^{*}} w=0$,

$\left(W_{b}+I\right) \mathrm{e}^{-A h^{*}} w=0$, it follows that $\mathrm{e}^{-A h^{*}} w$ is an eigenvector of $W_{a}$ corresponding to the eigenvalue 0 and an eigenvector of $W_{b}$ corresponding to the eigenvalue -1 .

Assume that $v$ is left eigenvector of $W_{a}$ corresponding to an eigenvalue $\lambda \neq 0$. Then,

$$
\begin{aligned}
v^{\mathrm{T}} W_{b} & =v^{\mathrm{T}} W_{a}-v^{\mathrm{T}} \mathrm{e}^{-A h^{*}} \frac{w C}{C w} \mathrm{e}^{A h^{*}} \\
& =v^{\mathrm{T}} W_{a}-\lambda^{-1} v^{\mathrm{T}} W_{a} \mathrm{e}^{-A h^{*}} \frac{w C}{C w} \mathrm{e}^{A h^{*}} \\
& =v^{\mathrm{T}} W_{a},
\end{aligned}
$$

where the last equality follows from Eq. (8). Hence, $v^{\mathrm{T}} W_{b}=\lambda v^{\mathrm{T}}$, so $\lambda$ is also an eigenvalue of $W_{b}$. Next, assume instead that $v$ is a left eigenvector of $W_{b}$ corresponding to an eigenvalue $\lambda \neq-1$. Then, similar to above,

$$
\begin{aligned}
v^{\mathrm{T}} W_{a} & =v^{\mathrm{T}} W_{b}+v^{\mathrm{T}} \mathrm{e}^{-A h^{*}} \frac{w C}{C w} \mathrm{e}^{A h^{*}} \\
& =v^{\mathrm{T}} W_{b}+(\lambda+1)^{-1} v^{\mathrm{T}}\left(W_{b}+I\right) \mathrm{e}^{-A h^{*}} \frac{w C}{C w} \mathrm{e}^{A h^{*}} \\
& =v^{\mathrm{T}} W_{b} \\
& =\lambda v^{\mathrm{T}}
\end{aligned}
$$

and the proof is complete.

Proposition 3 thus shows that if $C B=0$, the stability criteria in Propositions 1 and 2 are equivalent. Note, however, that Proposition 1 is applicable even if $C B \neq 0$.

Example 3. Consider the relay that feedback system in Example 1. Fig. 2 shows the function $f$ in Eq. (5) as

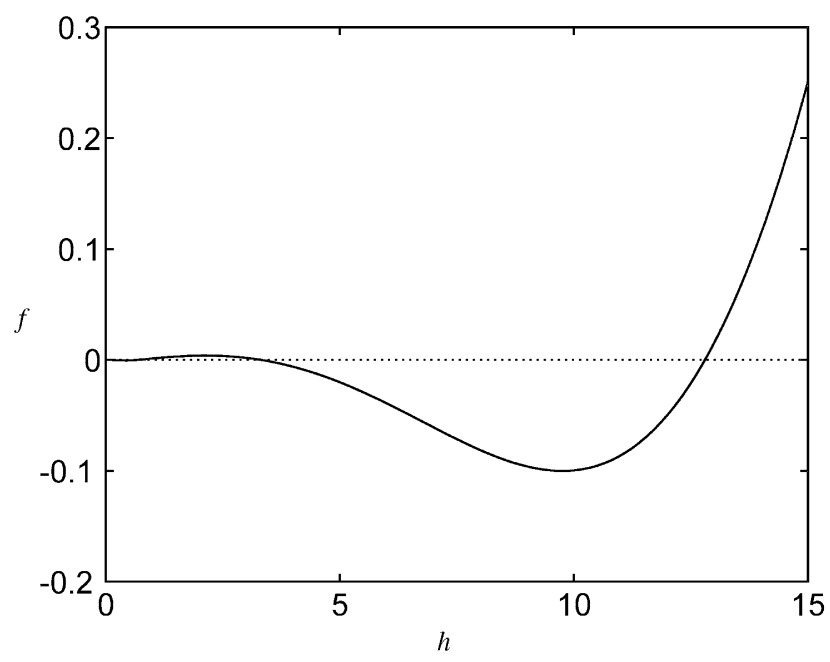

Fig. 2. The solutions of the equation $f(h)=0$, given in Eq. (5), yield possible limit cycle periods. For the system in Example 3 there exist three solutions (excluding $h=0$ ). 
a function of $h$. The zero-crossings for $h>0$ are at $0.66,3.32$, and 12.80 , so these are candidates for limit cycle periods. The eigenvalues with maximum magnitude of $W_{a}$ and $W_{b}$ (excluding the eigenvalue in -1 of $W_{b}$ ) for the three cases are $0.60,1.42$, and 0.64 , respectively. Only the first and the third zero-crossings thus come from a locally stable limit cycle. Note that we cannot draw any conclusions about global convergence.

\section{Existence of fast switches}

Relay switches with short switch times are studied in this section. A necessary and sufficient condition for a system to have consecutive fast relay switches is shown. Further insight is given in next section, where the dependency of the fast behavior on the pole excess is also investigated.

Let $h(\cdot)$ be the switch time as defined in Eq. (3). The relay feedback system (1)-(2) is said to have multiple fast switches near $x$ if for ever $\varepsilon>0$ there exists $x_{0} \in \mathscr{S}_{+}$such that $h\left(x_{0}\right)+h\left(g\left(x_{0}\right)\right)<\varepsilon$ and $\left|x_{0}-\hat{x}\right|<E$.

A sliding mode is the part of a trajectory that belongs to the switch plane: $x(t)$ is a sliding mode for $t \in\left(t_{1}, t_{2}\right)$ with $t_{1}>0$ if $C x(t)=0$ for all $t \in\left(t_{1}, t_{2}\right)$. Sliding modes are treated thoroughly in Filippov (1988). Let $r \in\{1, \ldots, n-1\}$ be the pole excess of $C(s I-A)^{-1} B$, so that $C A^{r-1} B \neq 0$ but $C A^{k} B=0$ for $k=0, \ldots, r-2$. Then the set $\left\{x: C x=C A x=\cdots=C A^{r-1} x=0\right\}$ is called the $r$ th-order sliding set, compare Fridman and Levant (1996). A sliding mode that belongs to an $r$ thorder sliding set is an $r$ th-order sliding mode.

The behavior of first-order sliding modes can easily be determined from studying $\dot{y}=C A x \pm C B$ close to the switch plane $\mathscr{S}$. We see that depending on the sign of $C B$, a classification of the directions of the trajectories divide the switch plane into two or three regions. We call the set $\mathscr{U}:=\{x \in \mathscr{S}:|C A x|<|C B|\}$ attractive, if for every $x \in \mathscr{U}$ there exists a neighborhood $\mathscr{B} \ni x$ such that every trajectory that starts in $\mathscr{B}$ stays there until it hits $\mathscr{U}$. We illustrate with a second-order example.

Example 4. Consider the same system as in Example 2, that is,

$G(s)=\frac{\beta s+1}{(s+1)(s+2)}$

with state-space representation

$\dot{x}=\left[\begin{array}{ll}0 & -2 \\ 1 & -3\end{array}\right] x+\left[\begin{array}{l}1 \\ \beta\end{array}\right] u$,

$y=\left[\begin{array}{ll}0 & 1\end{array}\right] x$,
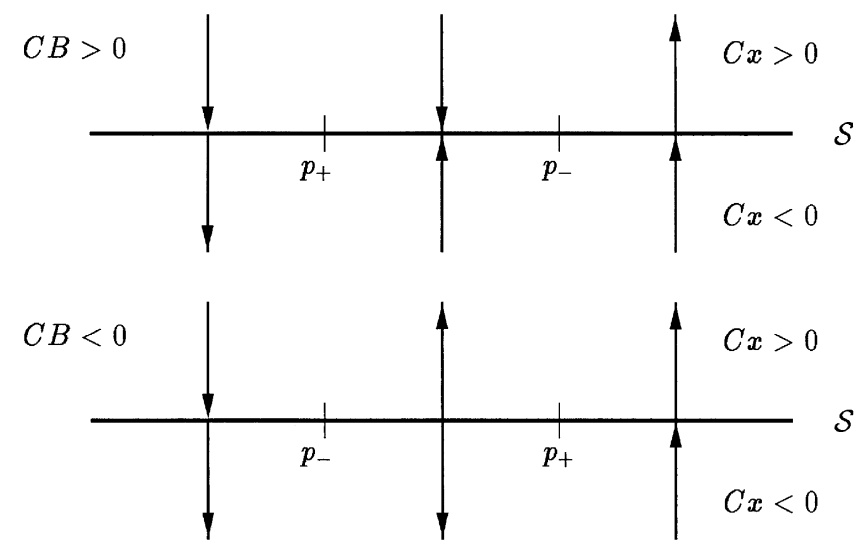

Fig. 3. Switch plane $\mathscr{S}$ and trajectories close to $\mathscr{S}$ for second-order system with $C B \neq 0$. The points $p_{+}$and $p_{-}$indicate where the trajectories change directions. The region between $p_{+}$and $p_{-}$is attractive if $C B>0$ but not if $C B<0$.

and relay feedback. Then $\mathscr{S}$ equals the $x_{1}$-axis, see Fig. 3 . Let $p_{+}, p_{-} \in \mathscr{S}$ be the solutions of the equations

$C A p_{+}+C B=0, \quad C A p_{-}-C B=0$,

respectively. These are the points where the trajectories change directions, and they are given by $p_{+}=(-\beta, 0)$ and $p_{-}=(\beta, 0)$. For $C B=\beta>0$ the set between $p_{+}$and $p_{-}$is attractive, whereas for $C B<0$ the region is repelling. The region vanishes if $C B=0$.

The condition in the example for the set $\{x \in \mathscr{S}$ : $|C A x|<|C B|\}$ to be attractive directly generalizes to systems of order $n>2$. Then $p_{+}$and $p_{-}$denote hyperplanes of dimension $n-2$, which still divide the switch plane into two or three regions. We can hence conclude that for the relay feedback system (1)-(2) with order $n \geq 2$ the set $\{x \in \mathscr{S}:|C A x|<|C B|\}$ is attractive if $C B>0$ but not if $C B<0$. We also notice that if $C B<0$ then the system does not have multiple fast switches.

Next, we consider systems with $C B=0$. Fig. 4 shows trajectories close to the second-order sliding set $\{x \in \mathscr{S}$ : $C A x=0\}$ for a third-order system with $C A B>0$ and $C A B<0$, respectively. The tick marks indicate solutions to the equations

$C A^{2} \chi_{-}-C A B=0, \quad C A^{2} \chi_{+}+C A B=0$,

that is, the points $x$ on the line $\{x \in \mathscr{S}: C A x=0\}$ such that the second derivative of the output $y$ is zero. Solid trajectories are above the switch plane $(C x>0)$ and dashed under $(C x<0)$. The figure suggests that consecutive switch times $h(\cdot)$ can be arbitrarily short if and only if $C A B>0$. A proof will be given next that for systems of arbitrary order to have multiple fast switches, it is necessary and sufficient for the first nonvanishing Markov parameter to be positive. 

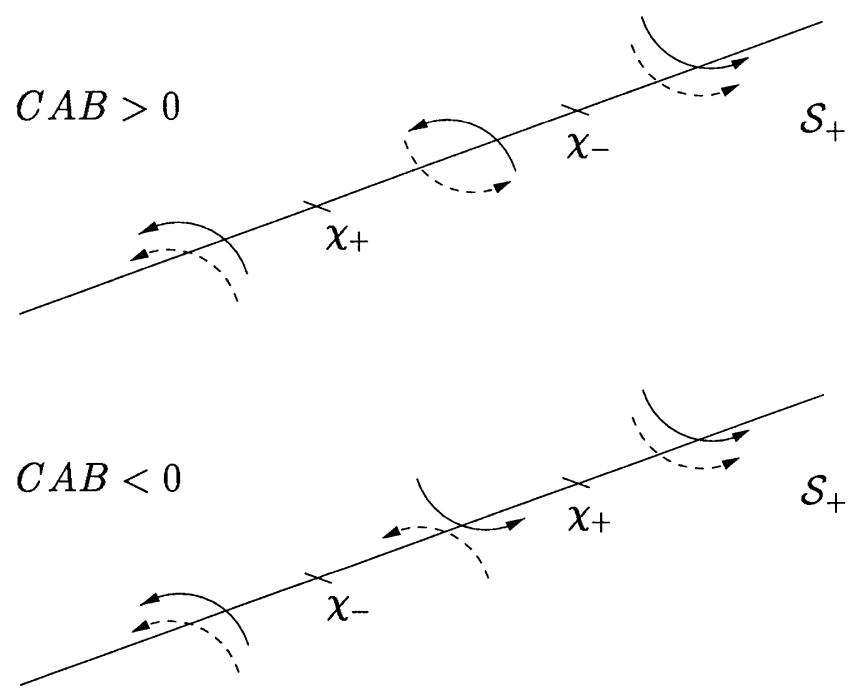

Fig. 4. The sign of the first non-vanishing Markov parameter determines the existence of multiple fast switches. Here the trajectories close to the second-order sliding set $\{x \in \mathscr{S}: C A x=0\}$ for a third-order system with $C B=0$ are shown. We have $C x>0$ above the switch plane and $C A x>0$ to the right of the line. Multiple fast switches occur if and only if $C A B>0$.

Theorem 1. Consider the relay feedback system (1)-(2) with order $n \geq 3$. Define $k \in\{1, \ldots, n-2\}$ such that $C A^{l} B=0$ for $l=0, \ldots, k-1$ and $C A^{k} B \neq 0$. The system has multiple fast switches if and only if $C A^{k} B>0$.

Proof. Let $\phi_{x}(t), t>0$, denote the trajectory of $\dot{x}=A x-B$ that starts in $x$ at time $t=0$. For $x \in \mathscr{S}_{+}$, Taylor expansion gives

$$
\begin{aligned}
C \phi_{x}(t)= & C A x t+\cdots+C A^{k} x \frac{t^{k}}{k !} \\
& +\left(C A^{k+1} x-C A^{k} B\right) \frac{t^{k+1}}{(k+1) !}+\mathcal{O}\left(t^{k+2}\right) .
\end{aligned}
$$

Sufficiency: Assume $C A^{k} B>0$. Then,

$C \phi_{0}\left(t_{0}\right)=-C A^{k} B \frac{t_{0}^{k+1}}{(k+1) !}+\mathcal{O}\left(t_{0}^{k+2}\right)<0$,

for $t_{0}>0$ sufficiently small. For a fixed such $t_{0}$, we have $C \phi_{\tilde{x}}\left(t_{0}\right)<0$ for all $\tilde{x} \in \mathscr{S}_{+}$with $|\tilde{x}|$ sufficiently small. Consider a fixed such $\tilde{x}$. Then, there exists a small $t \in\left(0, t_{0}\right)$ such that $C \phi_{x}(t)>0$, because $C A \tilde{x}>0$. In between $t$ and $t_{0}$ a switch thus occurs. Hence, we have that $h(x) \rightarrow 0$ as $x \rightarrow 0$ in $\mathscr{S}_{+}$and therefore also $g(x) \rightarrow 0$. The same type of argument gives that $h(g(x)) \rightarrow 0$.

Necessity: If the system has multiple fast switches, there exists a bounded sequence $\left\{x_{m}\right\}_{m=1}^{\infty}, x_{m} \in \mathscr{S}_{+}$, such that $h\left(x_{m}\right)+h\left(g\left(x_{m}\right) \rightarrow 0\right.$ as $m \rightarrow \infty$. After replacing $\left\{x_{m}\right\}_{m=1}^{\infty}$ with a suitable subsequene, we can assume that there exists $\hat{x} \in \mathscr{S}$ with $C A \hat{x}=0$ such that $x_{m} \rightarrow \hat{x}$. It is obvious that $g\left(x_{m}\right) \rightarrow-\hat{x}$. Now, assume $C A^{2} \hat{x}>0$. Then, there exists $t_{1}>0$ such that

$C \phi_{\hat{x}}(t)=C A^{2} \hat{x} \frac{t^{2}}{2}+\mathcal{O}\left(t^{3}\right)>0$,

for $t \in\left(0, t_{1}\right)$. Hence, $C \phi_{x_{m}}(t)>0$ for all $t \in\left(0, t_{1}\right)$ and $m$ sufficiently large. However, this contradicts that $h\left(x_{m}\right) \rightarrow 0$ as $m \rightarrow \infty$ and $C \phi_{x_{m}}\left(h\left(x_{m}\right)=0\right.$. Hence, $C A^{2} \hat{x} \leq 0$. A similar argument for $g\left(x_{m}\right)$ gives $C A^{2} \hat{x} \geq 0$, so we have $C A^{2} \hat{x}=0$. In the same way, $C A^{l} \hat{x}=0$ for every $l \in\{1, \ldots, k\}$. The same argument applied to term $k+1$ in Eq. (9) gives

$C A^{k+1} \hat{x}-C A^{k} B \leq 0, \quad C A^{k+1} g(\hat{x})-C A^{k} B \leq 0$,

or equivalently

$C A^{k+1} \hat{x} \leq C A^{k} B, \quad-C A^{k+1} \hat{x} \leq C A^{k} B$.

Hence, $C A^{k} B>0$ and the result follows.

Remark 1. It follows from the proof that multiple fast switches only occur close to $\left\{x \in \mathscr{S}: C A^{l} x=0\right.$, $l=1, \ldots, k\}$ in the region $\left|C A^{k+1} x\right|<C A^{k} B$.

The following example illustrates multiple fast switches in a third-order system.

Example 5. Consider the system

$G(s)=\frac{\zeta-s}{\zeta(s+1)^{3}}$

with state-space representation

$\dot{x}=\left[\begin{array}{lll}-3 & 1 & 0 \\ -3 & 0 & 1 \\ -1 & 0 & 0\end{array}\right] x+\left[\begin{array}{c}0 \\ -1 / \zeta \\ 1\end{array}\right] u$,

$y=\left[\begin{array}{lll}1 & 0 & 0\end{array}\right] x$,

and relay feedback. Fig. 5 shows two trajectories that start close to the origin for $\zeta=-4$ and $\zeta=1$, respectively. As predicted by Theorem 1, multiple fast switches occur when $C A B=-1 / \zeta>0$ but not when $C A B<0$. Compare Figs. 4 and 5.

The trajectories tend to a limit cycle for both systems. Fig. 6 shows the limit cycle period $2 h$ as a function of the zero $\zeta$. The dashed line corresponds to the limit cycle for the system $1 /(s+1)^{3}$. The origin is globally stable for the relay feedback system if $\zeta \in(-3,0)$, so in that case there will be no limit cycle. Local analysis around the limit cycle, as described in Section 3, gives in agreement with 

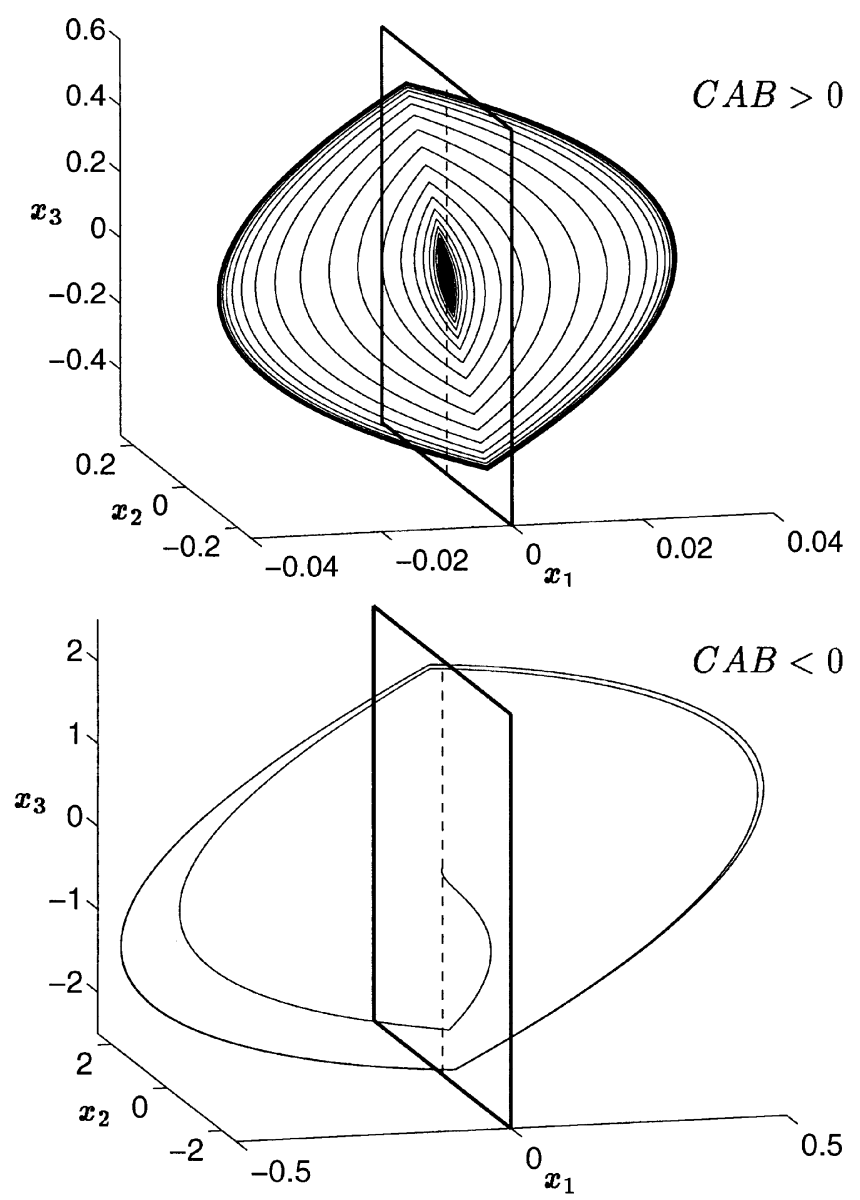

Fig. 5. Clockwise trajectories with initial conditions close to the origin for the third-order system in Example 5. Multiple fast switches exist if $C B=0$ and $C A B>0(\zeta<0)$. The system performs a large number of fast switches with slowly growing amplitude. If $C B=0$ and $C A B<0$ $(\zeta>0)$ there are no fast switches. Both trajectories converge to a limit cycle.

Fig. 5 that the convergence is faster if $\zeta=-4$ than if $\zeta=1$.

\section{Nature of fast switches}

Having established that the sign of the first nonvanishing Markov parameter determines if there will be multiple fast switches, we will now investigate the nature of the fast switches in more detail. It turns out that the behavior is given by the pole excess and one or two of the first nonvanishing Markov parameters. The first-order sliding set, which is present for systems with pole excess one, was discussed in connection to Example 4. In this section, we study the nature of fast switches for systems with pole excess two, pole excess three, and higher-order pole excess. Anosov (1959) showed that systems with pole excess two may have a large number of fast switches. He also showed that this is not the case for systems with pole excess greater than two.

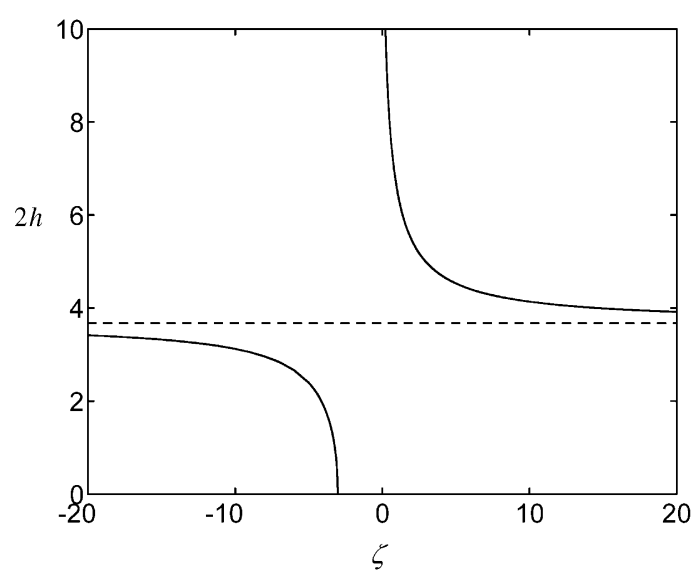

Fig. 6. The limit cycle period as a function of zero location in Example 5.

\subsection{Pole excess two - many fast switches}

There exist initial conditions that give a large number of fast switches if $C B=0$ and $C A B>0$. The fast response of a system with pole excess two is approximately described by a double integrator

$\dot{x}=\left[\begin{array}{ll}0 & 1 \\ 0 & 0\end{array}\right] x+\left[\begin{array}{l}0 \\ 1\end{array}\right] u$,

$y=\left[\begin{array}{ll}1 & 0\end{array}\right] x$.

Assume the trajectory of this system with relay feedback passes the switch plane at time $t=0$ at

$x(0)=\left[\begin{array}{ll}0 & x_{20}\end{array}\right]^{\mathrm{T}}, \quad x_{20}>0$.

Then, until next switch

$x_{1}(t)=x_{20} t-t^{2} / 2$,

$x_{2}(t)=x_{20}-t$.

The first equation gives that the first switch occurs at $h_{1}=2 x_{20}$. Between the first and the second switch we have

$x_{1}\left(h_{1}+t\right)=x_{20} t-h_{1} t+t^{2} / 2$,

$x_{2}\left(h_{1}+t\right)=x_{20}-h_{1}+t$,

so the second switch time is $h_{2}=2\left(h_{1}-x_{20}\right)=2 x_{20}$. Hence, $h_{k}=2 x_{20}$ for all $k>0$, so a double integrator with relay feedback has a limit cycle with any period. Next, consider the system

$G(s)=K / s(s+a), \quad K>0$,

and let the relay be approximated with a steep slope. Then, a root-locus argument predicts fast oscillations with increasing amplitude if $a<0$ and fast oscillations 
with decreasing amplitude if $a>0$. The double integrator with a neutral stable oscillation corresponds to $a=0$.

A higher-order system with zeros $\left\{z_{i}\right\}$, poles $\left\{p_{i}\right\}$, and pole excess two can be written as

$G(s)=K \frac{\prod_{i=1}^{n-2}\left(s-z_{i}\right)}{\prod_{i=1}^{n}\left(s-p_{i}\right)}=K \frac{\prod_{i=1}^{n-2}\left(1-z_{i} / s\right)}{s^{2} \prod_{i=1}^{n}\left(1-p_{i} / s\right)}$.

A series expansion in $1 / \mathrm{s}$ gives the terms that dominate the behavior of the system for high frequencies. Hence,

$G(s) \approx \frac{K}{s(s+a)}$,

where

$a=\sum_{i=1}^{n-2} z_{i}-\sum_{i=1}^{n} p_{i}$

The behavior of the system is thus governed by the sign of the parameter $a=C A^{2} B / K$. The oscillations are unstable for $a<0$, neutral for $a=0$, and damped for $a>0$. We illustrate with a simulation.

Example 6. Consider the system in Example 5:

$G(s)=\frac{\zeta-s}{\zeta(s+1)^{3}}$.

Here, $a=\zeta+3$. Fig. 7 shows the output $y$ for $a=-1,0,1$ and initial condition $x(0)$ close to the origin.

\subsection{Pole excess three - few fast switches}

Systems of pole excess higher than two cannot have fast oscillations as the ones shown in Fig. 7. A triple integrator represents the fast behavior in systems of pole excess three. Therefore, consider the system

$\dot{x}=\left[\begin{array}{lll}0 & 1 & 0 \\ 0 & 0 & 1 \\ 0 & 0 & 0\end{array}\right] x+\left[\begin{array}{l}0 \\ 0 \\ 1\end{array}\right] u$,

$y=\left[\begin{array}{lll}1 & 0 & 0\end{array}\right] x$,

with relay feedback. Assume the trajectory of the system passes the switch plane at time $t=0$ at

$x(0)=\left[\begin{array}{lll}0 & x_{20} & x_{30}\end{array}\right]^{\mathrm{T}}, \quad x_{20}>0$.

Then, until next switch

$x_{1}(t)=x_{20} t+x_{30} t^{2} / 2-t^{3} / 6$,

$x_{2}(t)=x_{20}+x_{30} t-t^{2} / 2$,

$x_{3}(t)=x_{30}-t$.

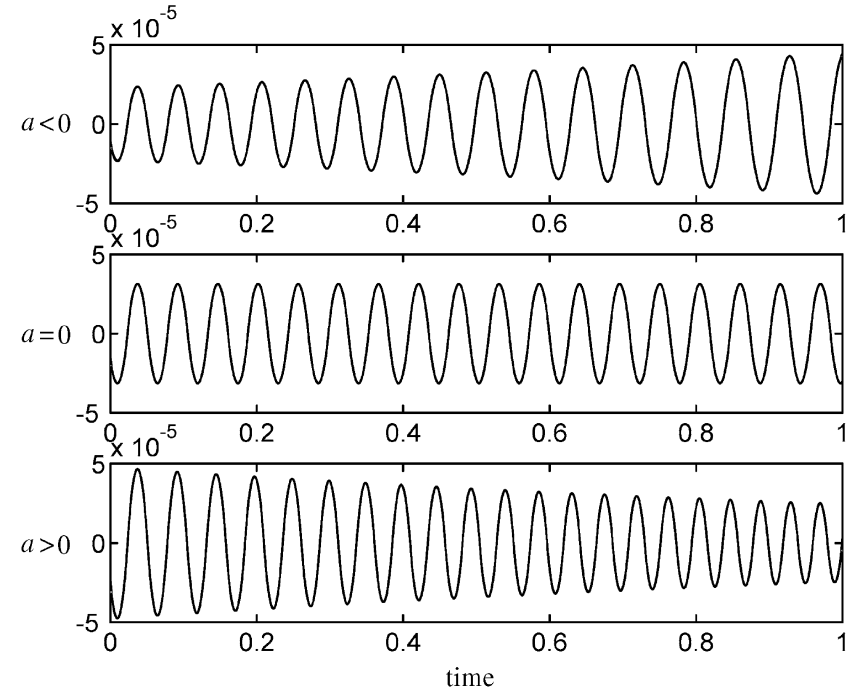

Fig. 7. Fast oscillations for systems with pole excess two. The oscillations are unstable, neutral, or damped, depending on the parameter $a$ in Eq. (10).

Because $x_{1}\left(h_{1}\right)=0$, the first switch time fulfills the equation $h_{1}^{2}-3 x_{30} h_{1}-6 x_{20}=0$.

Continued evaluation of the state-space system gives at the second switch instant, where $x_{1}\left(h_{1}+h_{2}\right)=0$, that

$h_{2}^{2}+3\left(x_{30}-h_{1}\right) h_{2}+6 x_{20}+6 x_{30} h_{1}-3 h_{1}^{2}=0$.

By solving for $x_{20}$ and $x_{30}$ in these two equations, we get

$x_{20}=h_{1} \frac{h_{2}^{2}-h_{1}^{2}-2 h_{1} h_{2}}{6\left(h_{1}+h_{2}\right)}$,

$x_{30}=\frac{2 h_{1}^{2}-h_{2}^{2}+3 h_{1} h_{2}}{3\left(h_{1}+h_{2}\right)}$.

Becausse $x_{20}>0$, we have $h_{2}^{2}-h_{1}^{2}-2 h_{1} h_{2}>0$ and thus $h_{2}>(1+\sqrt{2}) h_{1}$. Repeated evaluation yields

$h_{k}>(1+\sqrt{2})^{h-1} h_{1}$.

This estimate gets tighter as the initial state approaches the origin. We can conclude that there is a substantial increase in switch time after each interaction for a triple integrator.

Higher-order systems with pole excess three can be analyzed via a series expansion similar to the one in previous section. At high frequencies, these systems respond as a triple integrator:

$G(s) \approx K / s^{3}, \quad K>0$.

From a root-locus argument, we see that any fast behavior is unstable.

\subsection{Higher-order pole excess - fewer fast switches}

The increase in switch time after each fast relay switch is even higher for systems with pole excess larger than 
three. Consider an integrator of order $n$

$$
\begin{aligned}
\dot{x} & =\left[\begin{array}{ccccc}
0 & 1 & 0 & \cdots & 0 \\
0 & 0 & 1 & & 0 \\
\vdots & & & \ddots & \vdots \\
0 & 0 & 0 & & 1 \\
0 & 0 & 0 & \cdots & 0
\end{array}\right] x+\left[\begin{array}{c}
0 \\
0 \\
\vdots \\
0 \\
1
\end{array}\right] u, \\
y & =\left[\begin{array}{lllll}
1 & 0 & 0 & \cdots & 0
\end{array}\right] x,
\end{aligned}
$$

and introduce the partitioned matrices

$\left[\begin{array}{l|l}1 & \alpha(t) \\ \hline 0 & V(t)\end{array}\right]:=$

$\mathrm{e}^{A t}=\left[\begin{array}{c|cccc}1 & t & \frac{1}{2} t^{2} & \cdots & \frac{1}{(n-1) !} t^{n-1} \\ \hline 0 & 1 & t & \cdots & \frac{1}{(n-2) !} t^{n-2} \\ \vdots & & & \ddots & \vdots \\ 0 & 0 & 0 & & t \\ 0 & 0 & 0 & \cdots & 1\end{array}\right]$

and

$$
\left[\frac{\beta(t)}{\gamma(t)}\right]:=\int_{0}^{t} \mathrm{e}^{A \tau} B \mathrm{~d} \tau=\left[\begin{array}{c}
\frac{\frac{1}{n !} t^{n}}{\frac{1}{(n-1) !} !^{n-1}} \\
\vdots \\
\frac{1}{2} t^{2} \\
t
\end{array}\right] .
$$

Let the initial state

$x(0)=\left[\frac{0}{\xi^{0}}\right]$

lie on the switch plane and assume $\xi_{1}^{0}>0$, so that the trajectory passes through $\mathscr{S}_{+}$. Then, we have

$x(t)=\left[\frac{\alpha(t) \xi^{0}-\beta(t)}{V(t) \xi^{0}-\gamma(t)}\right], \quad 0<t<h_{1}$,

where $h_{1}$ is the first switch time. Hence,

$\alpha\left(h_{1}\right) \xi^{0}=\beta\left(h_{1}\right)$,

$\xi^{1}=V\left(h_{1}\right) \xi^{0}-\gamma\left(h_{1}\right)$.

Furthermore, for the second switch time $h_{2}$,

$\alpha\left(h_{2}\right) \xi^{1}=-\beta\left(h_{2}\right)$,

$\xi^{2}=V\left(h_{2}\right) \xi^{1}+\gamma\left(h_{2}\right)$,

so that

$\alpha\left(h_{2}\right) V\left(h_{1}\right) \xi^{0}=\alpha\left(h_{2}\right) \gamma\left(h_{1}\right)-\beta\left(h_{2}\right)$.
Continued evaluation gives

$$
\begin{aligned}
\alpha\left(h_{k}\right) & V\left(h_{k-1}\right) \cdots V\left(h_{1}\right) \xi^{0} \\
= & \alpha\left(h_{k}\right) V\left(h_{k-1}\right) \cdots V\left(h_{2}\right) \gamma\left(h_{1}\right) \\
& -\alpha\left(h_{k}\right) V\left(h_{k-2}\right) \cdots V\left(h_{3}\right) \gamma\left(h_{2}\right)+\cdots \\
& -(-1)^{k} \alpha\left(h_{k}\right) V\left(h_{k-1}\right) \gamma\left(h_{k-2}\right)+(-1)^{k} \alpha\left(h_{k}\right) \gamma\left(h_{k-1}\right) \\
& -(-1)^{k} \beta\left(h_{k}\right) .
\end{aligned}
$$

Stacking $n-1$ of these equations yields a linear equation in $\xi^{0}$. An analysis similar to the preceding for the triple integrator is therefore possible. It results in lower bounds on the switch times $h_{k}$. The analysis is particular simple if we assume the initial condition $\xi^{0}=\left(\xi_{1}^{0}, \ldots, 0\right)$. Then, Eq. (11) gives $h_{1} \xi_{1}^{0}=h_{1}^{n} / n !$ or $h_{1}=n !^{n-1} \sqrt{\xi_{1}^{0}}$.

Hence, for small initial states, the switch time increases considerably with the number of integrators $n$. Furthermore, Eq. (12) gives after some calculations

$\left(1+\frac{h_{1}}{h_{2}}\right)^{n}=2+\left(\frac{h_{1}}{h_{2}}\right)^{n-1}+\left(\frac{h_{1}}{h_{2}}\right)^{n}$.

Therefore, for $h_{1}$ much smaller than $h_{2}$, we have the formula

$h_{2} \approx(\sqrt[n]{2}-1)^{-1} h_{1}$.

Analysis that gives similar results can be done assuming other initial states $\xi^{0}$.

The fast behavior in systems with pole excess greater than or equal to three is thus unstable. The number of fast switches following a given initial state decreases with increasing pole excess.

\subsection{Summary}

The pole excess is important to characterize the solutions in relay feedback systems. With pole excess one there can be an attractive subset of the first-order sliding set. For the system $1 / s^{2}$ there will be limit cycles of arbitrary period. The limit cycles are not asymptotically stable. For systems of higher order with pole excess two, the behavior can be understood from a series expansion. In a similar way, the fast switches in any system of pole excess $k>0$ can be analyzed by studying an integrator of order $k$. There is, however, a fundamental difference between fast switches for systems with pole excess two and systems with pole excess three and higher. Only systems with pole excess one and two can have an attractive sliding set in the sense that an open set of trajectories tend to the sliding set. Note that the dimension of the sliding set is equal to the pole excess. 


\section{Fast switches in limit cycles}

Sliding modes and multiple fast switches can be part of stable limit cycles. Next we analyze these limit cycles and show some examples. It is the pole excess of the system that determines the kind of fast behavior the limit cycle will contain. The limit cycles presented here are further analyzed in Johansson (1997) and Johansson et al. (1997).

\subsection{Pole excess one - limit cycles with first-order sliding modes}

Consider a relay feedback system (1)-(2) with $C B>0$. The discussion in connection to Example 4 indicated that there exists an attractive subset of the first-order sliding set. The corresponding sliding mode can be part of a stable limit cycle.

Suppose that there exists a limit cycle and that the limit cycle consists of one "smooth" part, which is the part outside the switch plane, and one sliding mode part. Furthermore, suppose that the part outside the switch plane starts at time $t=0$ in $x^{0}=x(0)$ with $C A x^{0}=C B$. The trajectory of the system will then follow the dynamics $\dot{x}=A x-B$. Assume that the trajectory hits the switch plane at $t=t_{\mathrm{sm}}$ in $x^{1}=x\left(t_{\mathrm{sm}}\right)$ with $\left|C A x^{1}\right|<C B$, where $t_{\mathrm{sm}}$ is the time it takes for the smooth part. The sliding mode can then be derived in the following way. Replace $u$ in Eq. (1) with

$\bar{u}=-C A x / C B$,

such that $C \dot{x}=C A x+C B \bar{u}=0$. The variable $\bar{u}$ is called the equivalent control (Utkin, 1987). The dynamics of the sliding mode are given by

$\dot{x}=P A x$,

where

$P:=I-B C / C B$

is a projection matrix fulfilling $C P=0$ and $P B=0$. The projection is such that $C x(t)=0$ until $C A x(t)=-C B$. If the limit cycle is symmetric and simple, it leaves the switch plane at time $t_{\mathrm{sm}}+t_{\mathrm{sl}}$ at $x\left(t_{\mathrm{sm}}+t_{\mathrm{sl}}\right)=-x^{0}$, where $t_{\mathrm{sl}}$ is the time for the sliding mode part. We have the following necessary condition for the described limit cycle.

Proposition 4. Consider the relay feedback (1)-(2) with $C B>0$. If there exists a simple symmetric limit cycle with a first-order sliding mode and period time $2 h^{*}$, then

$$
\begin{aligned}
f_{1}\left(t_{\mathrm{sm}}, t_{\mathrm{sl}}\right):= & C\left(\mathrm{e}^{A t_{\mathrm{sm}}} \mathrm{e}^{P A t_{\mathrm{sl}}}+I\right)^{-1}\left(\mathrm{e}^{A t_{\mathrm{sm}}}-I\right) A^{-1} B=0, \\
f_{2}\left(t_{\mathrm{sm}}, t_{\mathrm{sl}}\right):= & C A\left(\mathrm{e}^{P A t_{\mathrm{s}}} \mathrm{e}^{A t_{\mathrm{sm}}}+I\right)^{-1} \mathrm{e}^{P A t_{\mathrm{s}}}\left(\mathrm{e}^{A t_{\mathrm{sm}}}-I\right) A^{-1} B \\
& +C B=0,
\end{aligned}
$$

where $h^{*}=t_{\mathrm{sm}}+t_{\mathrm{sl}}$. Here, $t_{\mathrm{sm}}$ is the time for the part of the trajectory outside the switch plane and $t_{\mathrm{sl}}$ is the sliding mode time.
Proof. Using the notation introduced above, we have that

$$
\begin{aligned}
& x^{1}=\mathrm{e}^{A t_{\mathrm{sm}}} x^{0}-\left(\mathrm{e}^{A t_{\mathrm{sm}}}-I\right) A^{-1} B, \\
& -x^{0}=\mathrm{e}^{P A t_{\mathrm{sl}}} x^{1} .
\end{aligned}
$$

Solving for $x^{1}$ gives

$x^{1}=\left(\mathrm{e}^{A t_{\mathrm{sm}}} \mathrm{e}^{P A t_{\mathrm{s}}}+I\right)^{-1}\left(\mathrm{e}^{A t_{\mathrm{sm}}}-I\right) A^{-1} B$,

so $f_{1}\left(t_{\mathrm{sm}}, t_{\mathrm{sl}}\right)=0$ follows from $C x^{1}=0$. Furthermore,

$$
\begin{aligned}
x^{0} & =-\mathrm{e}^{P A t_{\mathrm{s}}}\left(\mathrm{e}^{A t_{\mathrm{sm}}} \mathrm{e}^{P A t_{\mathrm{sl}}}+I\right)^{-1}\left(\mathrm{e}^{A t_{\mathrm{sm}}}-I\right) A^{-1} B \\
& =-\left(\mathrm{e}^{P A t_{\mathrm{s}}} \mathrm{e}^{A t_{\mathrm{sm}}}+I\right)^{-1} \mathrm{e}^{P A t_{\mathrm{s}}}\left(\mathrm{e}^{A t_{\mathrm{sm}}}-I\right) A^{-1} B,
\end{aligned}
$$

so $f_{2}\left(t_{\mathrm{sm}}, t_{\mathrm{sl}}\right)=0$ follows from $C A x^{0}=C B$.

Remark 2. The points where a limit cycle hits and leaves the switch plane are given by Eqs. (15) and (16), respectively.

Remark 3. Using $C x^{0}=0$ instead of $C x^{1}=0$ in the proof, gives an equivalent condition. This follows because $C \mathrm{e}^{P A t_{\mathrm{si}}}=C$ and thus

$C \mathrm{e}^{P A t_{\mathrm{s}}}\left(\mathrm{e}^{A t_{\mathrm{sm}}} \mathrm{e}^{P A t_{\mathrm{s}}}+I\right)^{-1}=C\left(\mathrm{e}^{A t_{\mathrm{sm}}} \mathrm{e}^{P A t_{\mathrm{sl}}}+I\right)^{-1}$.

The solutions of the equations $f_{1}\left(t_{1}, t_{2}\right)=0$ and $f_{2}\left(t_{1}, t_{2}\right)=0$ give candidates for switch times. This is illustrated in Exampe 7, compare Example 3 in Section 3.

To get some more insight, we adopt the state-space representation

$\dot{x}=\left[\begin{array}{ccccc}-a_{1} & 1 & 0 & \cdots & 0 \\ -a_{2} & 0 & 1 & & 0 \\ \vdots & & & \ddots & \vdots \\ -a_{n-1} & 0 & 0 & & 1 \\ -a_{n} & 0 & 0 & \cdots & 0\end{array}\right] x+\left[\begin{array}{c}1 \\ b_{2} \\ \vdots \\ b_{n-1} \\ b_{n}\end{array}\right] u$,

$y=\left[\begin{array}{lllll}1 & 0 & 0 & \cdots & 0\end{array}\right] x$,

where we normalized such that $C B=1>0$. Note that $C x=x_{1}$ and that $x \in \mathscr{S}$ implies $C A x=x_{2}$. Moreover,

$P A=\left[\begin{array}{ccccc}0 & 0 & 0 & \cdots & 0 \\ b_{2} a_{1}-a_{2} & -b_{2} & 1 & & 0 \\ \vdots & & & \ddots & \vdots \\ b_{n-1} a_{1}-a_{n-1} & -b_{n-1} & 0 & & 1 \\ b_{n} a_{1}-a_{n} & -b_{n} & 0 & \cdots & 0\end{array}\right]$, 
so the sliding dynamics

$\dot{z}=\left[\begin{array}{cccc}-b_{2} & 1 & \cdots & 0 \\ \vdots & & & \vdots \\ -b_{n-1} & 0 & & 1 \\ -b_{n} & 0 & \cdots & 0\end{array}\right] z$,

$z:=\left[\begin{array}{lll}x_{2} & \cdots & x_{n}\end{array}\right]^{\mathrm{T}}$

are unstable if and only if the polynomial

$b(s)=s^{n-1}+b_{2} s^{n-2}+\cdots+b_{n-1} s+b_{n}$

is unstable. The sliding mode time $t_{\mathrm{sl}}$ depends on the zeros of $b$.

Example 7. Consider

$G(s)=\frac{(s-\zeta)^{2}}{(s+1)^{3}}, \quad \zeta>0$

with state-space representation

$\dot{x}=\left[\begin{array}{lll}-3 & 1 & 0 \\ -3 & 0 & 1 \\ -1 & 0 & 0\end{array}\right] x+\left[\begin{array}{c}1 \\ -2 \zeta \\ \zeta^{2}\end{array}\right] u$,

$y=\left[\begin{array}{lll}1 & 0 & 0\end{array}\right] x$.

Then, $b(s)=(s-\zeta)^{2}$ has an unstable zero in $\zeta$. Let $\zeta=1$. The equations $f_{1}\left(t_{\mathrm{sm}}, t_{\mathrm{sl}}\right)=0$ and $f_{2}\left(t_{\mathrm{sm}}, t_{\mathrm{sl}}\right)=0$ in Proposition 4 have the solution $\left(t_{\mathrm{sm}}, t_{\mathrm{sl}}\right)=(4.04,0.39)$. This corresponds to

$x^{0}=\left[\begin{array}{l}0.00 \\ 1.00 \\ 3.35\end{array}\right], \quad x^{1}=\left[\begin{array}{c}0.00 \\ 0.47 \\ -3.42\end{array}\right]$,

and agrees with the simulated (clockwise) limit cycle shown in Fig. 8. The sliding dynamics are given by

$\dot{z}=\left[\begin{array}{cc}2 \zeta & 1 \\ -\zeta^{2} & 0\end{array}\right] z$

The sliding time $t_{\mathrm{sl}}$ decreases as $\zeta$ increasess.

There exists no stable system of lower order than three that gives a limit cycle with a first-order sliding mode. Stability of limit cycles with sliding modes are derived in Johansson (1997) and Johansson et al. (1997). For example, it is shown that the limit cycle in Example 7 is

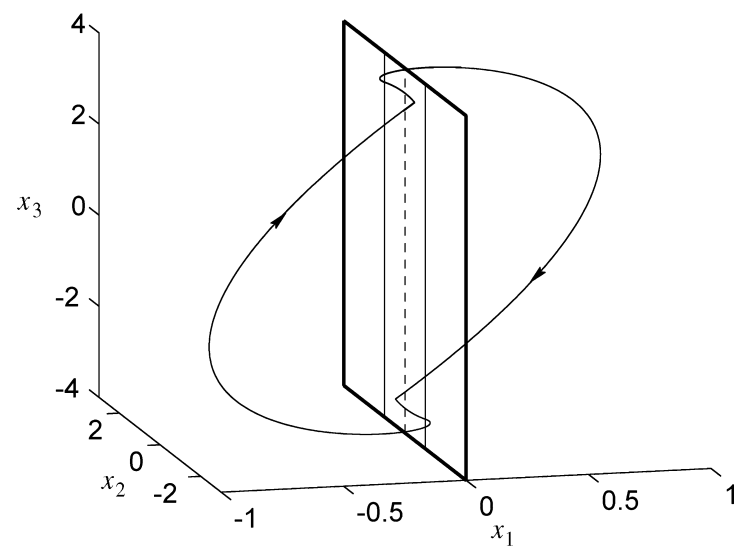

Fig. 8. Clockwise limit cycle with sliding mode. The dashed line in the switch plane illustrates the line $\{x \in \mathscr{S}: C A x=0\}$ and the solid lines illustrate $\{x \in \mathscr{S}:|C A x|=C B\}$. Note the points $\pm x^{0}$ and $\pm x^{1}$, where the limit cycle leaves and hits the switch plane, respectively.

stable. Limit cycles with sliding modes are also reported in Atherton et al. (1985) and Atherton (1993).

\subsection{Pole excess two - limit cycles with many fast switches}

Theorem 1 gives that systems with pole excess two have multiple fast switches if and only if $C A B>0$. Next, it is shown that these systems may have a limit cycle, where part of the limit cycle is such fast switches. The fast relay switches give a temporary fast oscillation in the state variables.

Let $C B=0$ and $C A B>0$. The second-order sliding mode can be derived by replacing $u$ in the original equation with $\bar{u}=-C A^{2} \bar{x} / C A B$. Then,

$\dot{x}=\left(I-\frac{B C A}{C A B}\right) A x$.

Adopting the state-space representation (17) but with

$B=\left[\begin{array}{lllll}0 & 1 & b_{3} & \cdots & b_{n}\end{array}\right]^{\mathrm{T}}$,

it is easy to see that this second-order sliding mode evolves in a subspace of dimension $n-2$. It is close to this subspace the fast oscillations, imposed by the fast relay switches, appear. The sliding mode is stable if the zeros of the linear system are stable. We have from Remark 1 of Theorem 1 that the fast behavior can only persist as long as $\left|C A^{2} x\right|<C A B$. Similar to the analysis of limit cycles with first-order sliding modes, the length of the time period with fast relay switches can be estimated. We illustrate with an example.

Example 8. Consider

$G(s)=\frac{(s-\zeta)^{2}}{(s+1)^{4}}, \quad \zeta>0$ 
with state-space representation

$\dot{x}=\left[\begin{array}{cccc}-4 & 1 & 0 & 0 \\ -6 & 0 & 1 & 0 \\ -4 & 0 & 0 & 1 \\ -1 & 0 & 0 & 0\end{array}\right] x+\left[\begin{array}{c}0 \\ 1 \\ -2 \zeta \\ \zeta^{2}\end{array}\right] u$,

$y=\left[\begin{array}{llll}1 & 0 & 0 & 0\end{array}\right] x$.

Let $\zeta=0.2$. Fig. 9 shows the limit cycle in the subspace $\left(x_{1} x_{2}, x_{3}\right)$. The previous sliding mode analysis gives that the fast oscillations should be close to the two-dimensional subspace $\left(x_{1}, x_{2}\right)$. This is illustrated in Fig. 10, where the fast oscillations around the line $\{x \in \mathscr{S}$ : $C A x=0\}=\left\{x: x_{1}=x_{2}=0\right\}$ are shown. Fig. 11 shows the four states during the fast switches. In agreement with the preceding analysis, the fast relay switches start when $C A^{2} x=-C A B$ and end when $C A^{2} x=C A B$, that is, at $x_{3}=-1$ and $x_{3}=1$, respectively. The state $x_{4}$ is approximately constant during the period of fast relay switches.

Formal conditions for stability of a limit cycle with fast switches as in Example 8 are given in Johansson (1997) and Johansson et al. (1997).

\subsection{Pole excess three - limit cycles with few fast switches}

The analysis done for systems of pole excess one and two also carries over to systems of higher-order pole excess. Next, we show an example of a system with pole excess three, which has a limit cycle with few extra switches each period.

Example 9. Consider

$G(s)=(s-\zeta)^{2} /(s+1)^{5}, \quad \zeta>0$

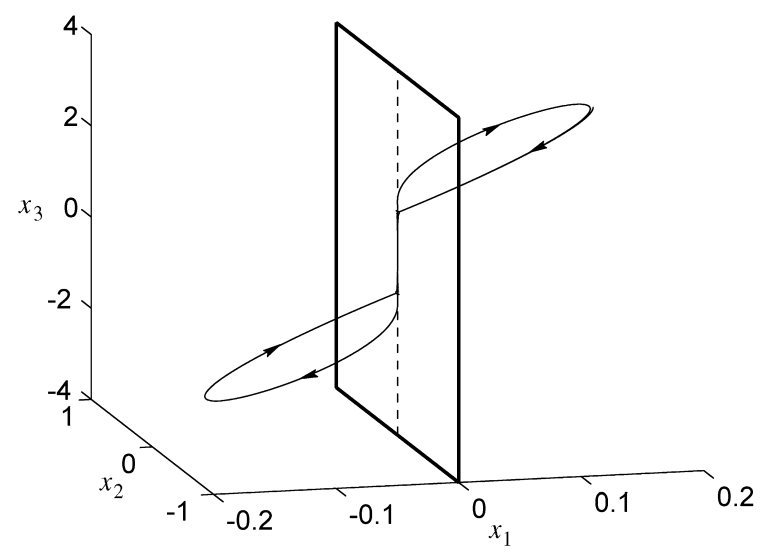

Fig. 9. Limit cycle with many fast relay switches for system with pole excess two. The two loops are clockwise. The dashed line in the switch plane illustrates the second-order sliding set $\{x \in \mathscr{S}: C A x=0\}$.

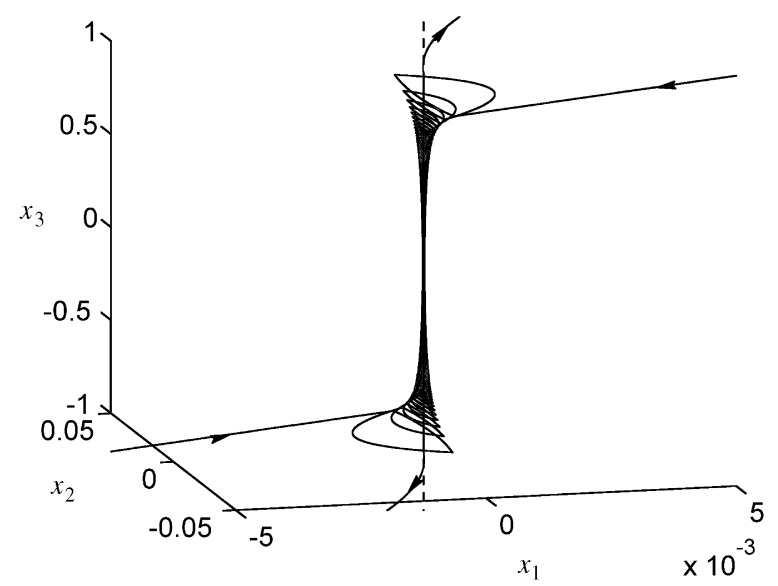

Fig. 10. A closer look at the fast oscillations in the limit cycle. The dashed line is the second-order sliding set $\{x \in \mathscr{S}: C A x=0\}$.
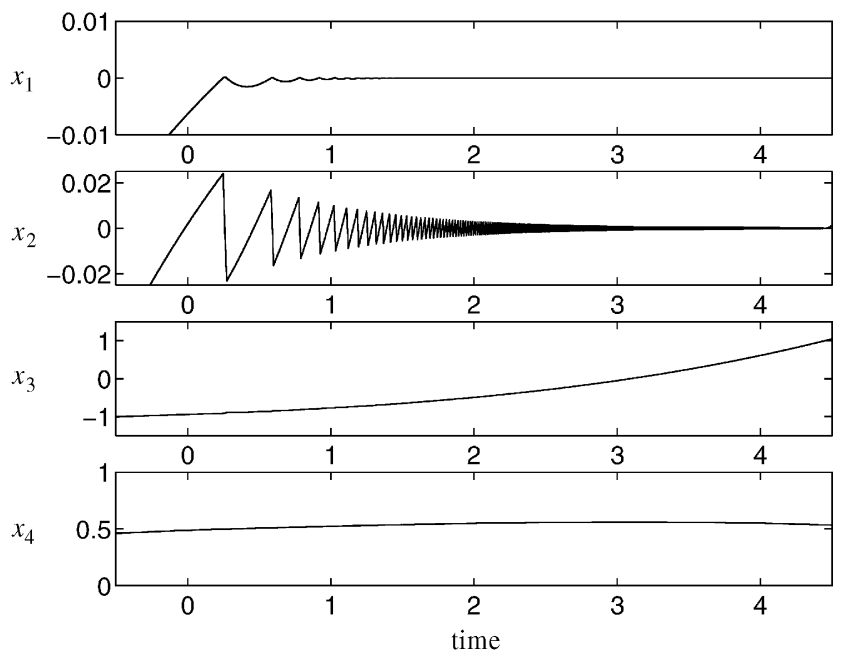

Fig. 11. Fast relay switches in a limit cycle for system with pole excess two. The fast oscillations start at $x_{3}=-1$ and end at $x_{3}=1$.

with state-space representation

$$
\dot{x}=\left[\begin{array}{ccccc}
-5 & 1 & 0 & 0 & 0 \\
-10 & 0 & 1 & 0 & 0 \\
-10 & 0 & 0 & 1 & 0 \\
-5 & 0 & 0 & 0 & 1 \\
-1 & 0 & 0 & 0 & 0
\end{array}\right] x+\left[\begin{array}{c}
0 \\
0 \\
1 \\
-2 \zeta \\
\zeta^{2}
\end{array}\right] u
$$

$y=\left[\begin{array}{lllll}1 & 0 & 0 & 0 & 0\end{array}\right] x$.

Let $\zeta=0.12$ and $x(0)=(0,1,0,0,0)^{\mathrm{T}}$. The convergence to the stable limit cyle is complicated as shown in Figs. 12 and 13 .

The limit cycle characteristics can, however, be predicted also in this case. Fig. 14 shows $x_{1}, \ldots, x_{4}$ during the limit cycle. Because the pole excess is three, there will 

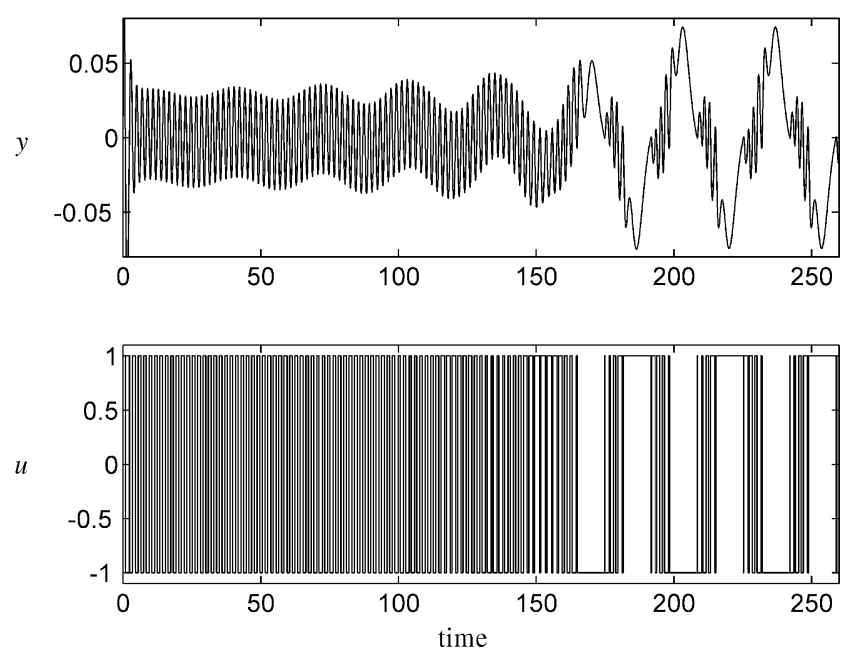

Fig. 12. Convergence to a limit cycle for a system with pole excess three. The output $y=x_{1}$ of the linear system and the output $u$ of the relay are shown.

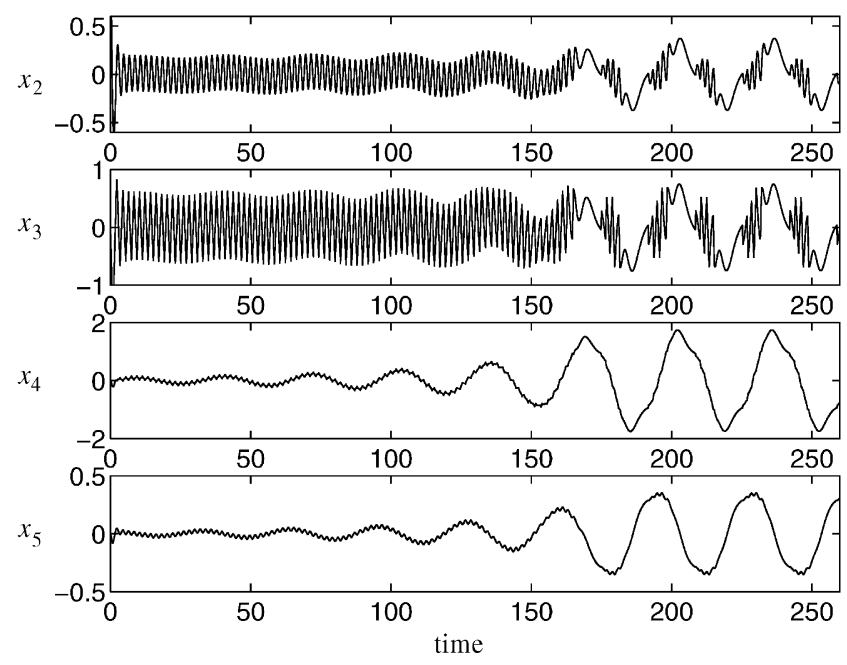

Fig. 13. The state variables $x_{2}, \ldots, x_{5}$ converging to a limit cycle for a system with pole excess three.

not be a large number of fast relay switches. Only eighteen switches occur each period of the limit cycle. Note, as we may expect, it is the points where $\left|C A^{3} x\right|=C A^{2} B$ that determines when the fast switching starts and ends. In this example they correspond to $x_{4}= \pm 1$. The state $x_{5}$ is approximately constant during the period of extra relay switches.

\section{Conclusions}

The problem of characterizing behaviors in relay feedback systems has been addressed. It was motivated by a number of examples from the literature, where one was the automatic tuning procedure for PID controllers using relay feedback by Åström and Hägglund. Another mo-

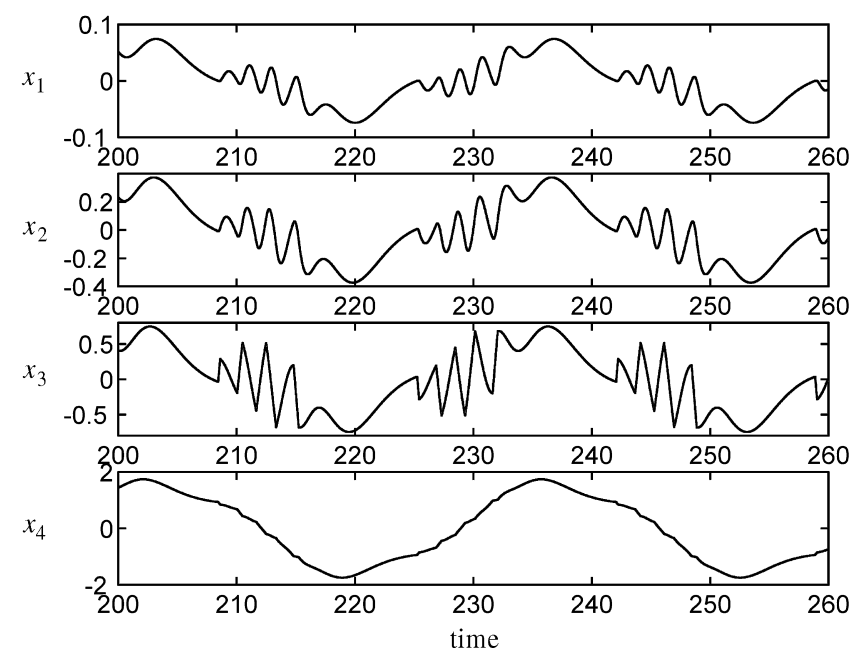

Fig. 14. A few fast switches occur each period of the limit cycle. These start and stop at $x_{4}= \pm 1$, that is, when $\left|C A^{2} x\right|=C A^{2} B$. (The state $x_{5}$ is not shown here, but it is approximately constant during the fast switches.)

tivation for the study of relay feedback systems is their connection to hybrid systems (Morse, 1995). The system we have considered can be viewed as a simple hybrid system that consists of only one discrete state.

The main result of the paper was a complete characterization of all relay feedback systems that have multiple fast switches. It was shown that multiple fast switches exist if and only if the first nonvanishing Markov parameter is positive. The nature of the fast behavior was further investigated. It was shown that there is a fundamental difference between systems of pole excess one, pole excess two, and pole excess greater than two. The fast behavior of these systems can be studied via relay feedback of an integrator, double integrator, and a higher-order integrator. The results on fast switches were applied to analysis of limit cycles, where part of the limit cycle consists of a sliding mode or a number of fast switches. Ongoing work includes stability analysis of these limit cycles (Johnasson et al., 1997). Local stability analysis of limit cycles without fast switches was also done. It was proved that two conditions in the literature are equivalent in most cases.

\section{Acknowledgements}

This research was supported by the Swedish Research Council for Engineering Science under contract 95-759.

\section{References}

Andronov, A. A., Khaikin, S. E., \& Vitt, A. A. (1965), Theory of oscillators. Oxford: Pergamon Press.

Anosov, D. V. (1959). Stability of the equilibrium positions in relay systems. Automat. Remote Control, 20, 135-149. 
Åström, K. J. (1995). Oscillations in systems with relay feedback. In Åström et al. (Eds.) Adaptive control, filtering, and signal processing IMA Volumes in Mathematics and its Applications (Vol. 74, pp. 1-25). Berlin: Springer.

Åström, K. J., \& Hägglund, T. (1984a). Automatic tuning of simple regulators. In Preprints 9th IFAC World Congress (pp. 267-272). Budapest, Hungary.

Åström, K. J., \& Hägglund, T. (1984b). Automatic tuning of simple regulators with specifications on phase and amplitude margins. Automatica, 20, 645-651.

Åström, K. J., \& Hägglund, T. (1995). PID controllers: Theory, design, and tuning (2nd ed.). Research Triangle Park, NC: Instrument Society of America.

Atherton, D. P. (1975). Nonlinear control engineering: Describing function analysis and design. London, UK: Van Nostrand Reinhold Co.

Atherton, D. P. (1993). Analysis and design of relay control systems. In Linkens (Ed.), CAD for control systems (Chapter 15, pp. 367-394). New York: Marcel Dekker.

Atherton, D. P., McNamara, O. P., \& Goucem, A. (1985). SUNS: The Sussex University nonlinear control systems software. In Preprints CADCE. Copenhagen.

Balasubramanian, R.(1981). Stability of limit cycles in feedback systems containing a relay. IEE Proc. D, 128(1), 24-29.

Filippov, A. F. (1988). Differential equations with discontinuous righthand sides. Dordrecht: Kluwer Academic Publishers.

Flügge-Lotz, I. (1953). Discontinuous automatic control. Princeton, NJ: Princeton University Press.

Flügge-Lotz, I. (1968). Discontinuous and optimal control. New York: McGraw-Hill.

Fridman, L. M., \& Levant A. (1996). Higher order sliding modes as a natural phenomenon in control theory. In Garofalo, \& Glielmo (Eds.), Robust control via variable structure \& Lyapunov techniques, Lecture notes in control and information science, Vol. 217 (pp. 107-133). Berlin: Springer.

Holmberg, U. (1991). Relay feedback of simple systems. Ph.D. thesis TFRT-1034, Department of Automatic Control, Lund Institute of Technology, Lund, Sweden.

Johansson, K. H. (1997). Relay feedback and multivariable control. Ph.D. thesis, TFRT-1048, Department of Automatic Control, Lund Institute of Technology, Lund, Sweden.

Johansson, K. H., Barabanov, A., \& Åström, K. J. (1997). Limit cycles with chattering in relay feedback systems. Proc. 36th IEEE Conference on Decision and Control, San Diego, CA.

Johansson, K. H., \& Rantzer, A. (1996). Global analysis of third-order relay feedback systems. In Preprints IFAC 13th World Congress (Vol. E, pp. 55-60). San Francisco, CA.

Lundh, M., \& Åström, K. J. (1994). Automatic initialization of a robust self-tuning controller. Automatica, 30(11), 1649-1662.

Mees, A. I. (1981). Dynamics of feedback systems. New York: Wiley.

Megretski, A. (1996). Global stability of oscillations induced by a relay feedback. In Preprints IFAC 13th Congress (Vol. E., pp. 49-54). San Francisco, CA.

Morse, A. S. (1995). Control using logic-based switching. In Isidori (Ed.), Trends in control. A European perspective (pp. 69-113). Berlin: Springer.

Parker, S. R., \& Hess, S. F. (1971). Limit cycle oscillations in digital filters. IEEE Trans. Circuits Theory, CT-18, 687-697.

Shevitz, D., \& Paden, B. (1994). Lyapunov stability theory of nonsmooth systems. IEEE Trans. Automat. Control, 39(9), 1910-1914.

Smith, R. S., \& Doyle, J. C. (1993). Closed loop relay estimation of uncertainty bounds for robust control models. In Preprints 12th IFAC World Congress (Vol. 9, pp. 57-60). Sydney, Australia.

Tsypkin, Ya. Z. (1984). Relay control systems. Cambridge, UK: Cambridge University Press.

Utkin, V. I. (1987). Discontinuous control systems: State of the art in theory and applications. In Preprints 10th IFAC World Congress. Munich, Germany.

Wadey, M. D., \& Atheron, D. P. (1986). A simulation study of unstable limit cycles. In IFAC Simulation of Control Systems (pp. 149-154). Vienna, Austria.
Yakubovich, V. A. (1964). The absolute stability of nonlinear control systems in critical cases. III. Automat. Remote Control, 25(5), 601-612.

Yakubovich, V. A. (1973). Frequency-domain criteria for oscillation in nonlinear systems with one stationary nonlinear component. Sibirsk. Mat. Zh., 14(5), 1100-1129.

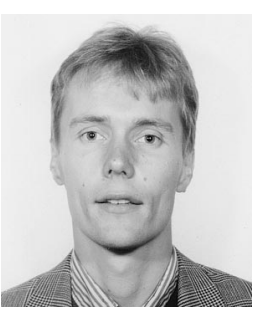

Karl Henrik Johansson was born in 1967. He received the M.S. degree in Electrical Engineering 1992 and the Ph.D. degree in Control Engineering 1997, both from Lund Institute of Technology, Sweden. He currently holds a postdoctoral position at the Department of Electrical Engineering and Computer Sciences, University of California at Berkeley.

Dr. Johansson's research interests are in switched control systems, multivariable control, relay controller tuning, and vari-

ous applications of control.

Dr. Johansson received the Peccei Award 1993 from the International Institute of Applied Systems Analysis in Austria and the Scania Research Award 1996 from Scania AB in Sweden. At the IFAC World Congress in 1996 he was awarded the Young Author Prize (together with Dr A. Rantzer).

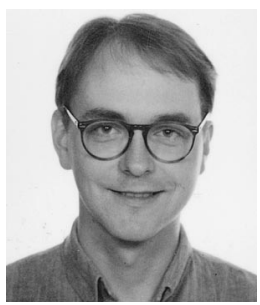

Anders Rantzer was born in 1963. He received a M.S. degree in Engineering Physics and a Licentiate degree in Mathematics, both from Lund Institute of Technology, Lund, Sweden, and a Ph.D. degree in Optimization and Systems Theory from the Royal Institute of Technology, Stockholm, Sweden. After post-doctoral positions at KTH and at IMA, University of Minnesota, he joined the Department of Automatic Control, Lund, in 1993.

Dr. Rantzer serves as Associate Editor of IEEE Transactions on Automatic Control, European Journal of Control and Systems and Control Letters. He is a winner of 1990 SIAM Student Paper Competition and 1996 IFAC World Congress Young Author Prize.

His research interests are in modeling, analysis and design of control systems, with particular attention to the effects of uncertainty and nonlinearities.

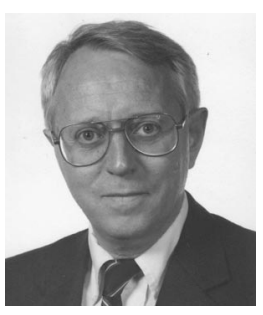

Karl Johan Åström is Professor and Head of the Department of Automatic Control at Lund University since 1965 . He has broad interests in Automatic Control including Stochastic Control, System Identification, Adaptive Control, Computer Control and Computer-aided Control Engineering. He has supervised $44 \mathrm{Ph}$.D. students, written six books and more than 100 papers in archival journals. He is a member of the Royal Swedish Academy of Engineering Sciences (IVA) and the Royal Swedish Academy of Sciences (KVA) and a foreign member of the US National Academy of Engineering and the Russian Academy of Sciences. Åström has received many honors including three honorary doctorates, the Quazza medal from IFAC, the Rufus Oldenburger Metal from ASME, the IEEE Control Systems Science Award and the IEEE Medal of Honor. 